Pacific

Journal of

Mathematics

SMOOTHNESS OF ALGEBRAIC SUPERVARIETIES

AND SUPERGROUPS

RITA FIORESI 


\title{
SMOOTHNESS OF ALGEBRAIC SUPERVARIETIES AND SUPERGROUPS
}

\author{
RITA FIORESI
}

\begin{abstract}
We discuss the notion of smoothness in complex algebraic supergeometry and prove that all affine complex algebraic supergroups are smooth. We then prove the stabilizer theorem in the algebraic context, providing some useful applications.
\end{abstract}

\section{Introduction}

The category of differentiable supermanifolds was introduced and discussed in several works - among which [Batchelor 1984; 1985; Berezin 1987; Kostant 1977; Leites 1980; Manin 1988] — from different point of views, especially in connection with important physical applications stemming from string theory and ultimately related to the problem of classification of elementary particles.

In this paper we are interested in algebraic supergeometry and its relation with its differential counterpart. In his foundational work on supermanifolds, Manin [1988] defined the notion of a superscheme and discussed some important examples.

Along the same lines we want to understand the concept of smoothness in complex algebraic supergeometry. Given the algebraic nature of the problems in the theory of supermanifolds, we believe that a deep analysis of the superalgebraic category can shed light also on the differential one. Moreover this is the correct category to work with when one wants to discuss quantum deformations of the geometric objects.

In ordinary algebraic geometry smoothness is a local notion, strongly linked to the dimension of the local ring of the variety at the point. Unfortunately, due to the presence of the odd nilpotents, it is not easy to generalize the idea of dimension of a ring to the supergeometric context. To overcome this problem, we define smoothness as a property of the completion of the local superring of the supervariety at a given point: a point is smooth if the local superring is isomorphic to a power series superring. We are then able to show that any supervariety admits a unique supermanifold structure in a neighborhood of a smooth point, as in the classical

MSC2000: 17B10.

Keywords: supervarieties, supergroups. 
case, through the application of the implicit function theorem, after reduction to local complete intersection.

Using Cartier's Theorem adapted to supergeometry we can then prove that all algebraic supergroups are smooth; in other words, all affine algebraic supergroups are also Lie supergroups. We apply this to the stabilizer supergroup functor of an action of an affine supergroup on an affine supervariety. After showing that the stabilizer is representable, hence a supergroup, we show that classical supergroups are smooth. ${ }^{1}$ This fact is generally known; it is treated in a different context in [Gruson 1994] and [Varadarajan 2004, p. 289], for example. However, we provide an independent proof using algebraic techniques, which we believe can be of help to other questions on differentiable supermanifold and may yield other examples of algebraic Lie supergroups.

This paper is organized as follows:

In Section 2, we review basic facts of algebraic and differential supergeometry, such as the definition of supermanifolds, supervarieties and their functor of points.

In Section 3 we define smooth points of a supervariety. We then prove the super version of the classical result that a smooth point of a complex algebraic variety admits a supermanifold structure in a suitable neighborhood.

In Section 4 we prove that all (closed) points of complex algebraic groups are smooth.

In Section 5 we prove the Stabilizer Theorem, which states that the stabilizer functor for the action of an affine algebraic supergroup on an affine supervariety is representable by a supergroup, so it is a smooth variety, hence a supermanifold.

As an application, in Section 6, we show that the classical supergroup functors as described in [Deligne and Morgan 1999, p. 70] are representable, and consequently they are Lie supergroups.

\section{Basic definitions of supergeometry}

In this section we recall some basic definitions and facts in supergeometry. For more details see [Varadarajan 2004; Caston and Fioresi 2007; Deligne and Morgan 1999; Manin 1988].

Let $k$ be the ground field. A superalgebra $A$ is a $\mathbb{Z}_{2}$-graded algebra, $A=A_{0} \oplus A_{1}$. We denote by $p(x)$ the parity of an homogeneous element $x \in A$. We say that $A$ is commutative if

$$
x y=(-1)^{p(x) p(y)} y x .
$$

Definition 2.1. A superspace $S=\left(|S|, O_{S}\right)$ is a topological space $|S|$ endowed with a sheaf of superalgebras $O_{S}$ such that the stalk $O_{S, x}$ is a local superalgebra

\footnotetext{
${ }^{1}$ For a list of classical supergroups see [Deligne and Morgan 1999, p. 70], for instance.
} 
for all $x \in|S|$. A morphism $\phi: S \rightarrow T$ of superspaces is given by $\phi=\left(|\phi|, \phi^{*}\right)$, where $\phi:|S| \rightarrow|T|$ is a map of topological spaces and $\phi^{*}: \mathbb{O}_{T} \rightarrow \phi^{*} \mathscr{O}_{S}$ is a sheaf morphism such that $\phi_{x}^{*}\left(m_{|\phi|(x)}\right)=m_{x}$ where $m_{|\phi|(x)}$ and $m_{x}$ are the maximal ideals in the stalks $\mathrm{O}_{T,|\phi|(x)}$ and $\mathrm{O}_{S, x}$ respectively.

The most important examples of superspaces are given by supermanifolds and superschemes.

Definition 2.2. Consider the superspace $\mathbb{C}^{p \mid q}=\left(\mathbb{C}^{p}, \mathcal{H}_{\mathbb{C}^{p \mid q}}\right)$, where

$$
\left.\mathscr{H}_{\mathbb{C} p \mid q}\right|_{U}=\left.\mathscr{H}_{\mathbb{C}^{p}}\right|_{U} \otimes \mathbb{C}\left[\xi_{1}, \ldots, \xi_{q}\right], \quad U \text { open in } \mathbb{C}^{p}
$$

where $\mathbb{C}\left[\xi_{1}, \ldots, \xi_{q}\right]$ is the exterior algebra generated by $\xi_{1}, \ldots, \xi_{q}$ and $\mathscr{H}_{\mathbb{C}^{p}}$ denotes the sheaf of holomorphic functions on $\mathbb{C}^{p}$.

A complex supermanifold of dimension $p \mid q$ is a superspace $M=\left(|M|, \mathscr{H}_{M}\right)$ which is locally isomorphic to $\mathbb{C}^{p \mid q}$; that is, for all $x \in|M|$ there exist open sets $V_{x} \subset|M|, U \subset \mathbb{C}^{p}$ such that

$$
\left.\mathrm{O}_{M}\right|_{V_{x}} \cong \mathscr{H}_{\left.\mathbb{C}^{p \mid q}\right|_{U}}
$$

Definition 2.3. A superscheme $S$ is a superspace $\left(|S|, \mathcal{O}_{S}\right)$ such that $\left(|S|, \mathcal{O}_{S, 0}\right)$ is a quasicoherent sheaf of $\mathrm{O}_{S, 1}$-modules. A morphism of supermanifolds or of superschemes is a morphisms of the corresponding superspaces.

Superschemes can be characterized by a local model:

Definition 2.4 ( $\operatorname{Spec} A)$. Let $A$ be a superalgebra and let $0_{A_{0}}$ be the structural sheaf of the ordinary scheme $\underline{\operatorname{Spec}}\left(A_{0}\right)=\left(\operatorname{spec} A_{0}, O_{A_{0}}\right)$ ( $\operatorname{spec} A_{0}$ denotes the prime spectrum of the commutative ring $A_{0}$ ). The stalk of the sheaf at the prime $p \in$ spec $A_{0}$ is the localization of $A_{0}$ at $p$. As for any superalgebra, $A$ is a module over $A_{0}$. We have indeed a sheaf $\mathrm{O}_{A}$ of $\mathrm{O}_{A_{0}}$-modules over spec $A_{0}$ with stalk $A_{p}$, the localization of the $A_{0}$-module $A$ over the prime $p \in \operatorname{spec} A_{0}$ :

$$
A_{p}=\left\{f / g \mid f \in A, g \in A_{0}-p\right\} .
$$

$A_{p}$ contains a unique two-sided maximal ideal generated by the maximal ideal in the local ring $\left(A_{p}\right)_{0}$ and the generators of $\left(A_{p}\right)_{1}$ as $A_{0}$-module.

$\mathrm{O}_{A}$ is a sheaf of superalgebras and ( $\left.\operatorname{spec} A_{0}, \mathrm{O}_{A}\right)$ is a superscheme that we denote with $\underline{\operatorname{Spec}} A$.

The next proposition shows that $\underline{\operatorname{Spec}} A$ is the local model for superschemes.

Proposition 2.5. A superspace $S$ is a superscheme if and only if it is locally isomorphic to Spec A for some superalgebra $A$; that is, for all $x \in|S|$, there exists $U_{x} \subset|S|$ open such that $\left(U_{x},\left.O_{S}\right|_{U_{x}}\right) \cong$ Spec $A$. (Clearly A depends on $U_{x}$.)

Proof. See [Caston and Fioresi 2007, §3]. 
Definition 2.6. We say that a superscheme $X$ is affine if it is isomorphic to $\underline{\operatorname{Spc}} A$ for some algebra $A$ and we call $k[X]:=A$ the coordinate ring of the affine superscheme $X$. Let $I^{\text {odd }}$ be the ideal generated by the odd nilpotents of $A$. If $k[X] / I^{\text {odd }}$ is the coordinate ring of an ordinary affine algebraic variety (called the reduced variety associated to $X)$ and $\left(|X|, O_{X, 0}\right)$ is a coherent sheaf of $O_{X, 1}$-modules, we say that $X$ is an affine algebraic variety.

Remark 2.7. There is an equivalence of categories between superalgebras and affine superschemes. This equivalence is treated in detail in [Caston and Fioresi 2007, §3].

Definition 2.8. Let $X$ be a supervariety. The functor of points of $X$ is given by

$$
h_{X}:(\operatorname{salg}) \rightarrow(\text { sets }), \quad h_{X}(A)=\operatorname{Hom}(\underline{\operatorname{Spec}} A, X),
$$

where (salg) is the category of commutative superalgebras. If $X$ is an affine supervariety $h_{X}(A)=\operatorname{Hom}(k[X], A)$. If $h_{X}$ is group-valued we say that $X$ is an affine supergroup. This is equivalent to the fact that $k[X]$ is a Hopf superalgebra. This is also the same as giving a multiplication $m: X \times X \rightarrow X$ and an inverse $i: X \rightarrow X$ satisfying the usual commutative diagrams.

More generally, we say that $G:$ (salg) $\rightarrow$ (sets) is a supergroup functor if it is group-valued. Clearly, a representable supergroup functor is an affine supergroup.

\section{Smoothness of complex algebraic supervarieties}

Let $k=\mathbb{C}$.

Let $X=\left(|X|, O_{X}\right)$ be a supervariety and let $P \in|X|$ be a closed point; that is, $P$ corresponds to a maximal ideal. Let $m_{P}$ be the maximal ideal in $\hat{O}_{X, P}$.

Definition 3.1. We say that $P$ is smooth if

$$
\widehat{\mathrm{O}_{X, P}} \cong \mathbb{C} \llbracket x_{1}, \ldots, x_{r}, \xi_{1}, \ldots, \xi_{s} \rrbracket, \quad \widehat{\mathrm{O}_{X, P}}=\lim _{\leftarrow} \widehat{O}_{X, P} / m_{P}^{n}
$$

where the $x_{i}$ and $\xi_{j}$ are respectively even and odd variables. In this case we say that the dimension of the supervariety $X$ at $P$ is $r \mid s$. Notice that the dimension is well defined, that is if $\mathbb{C}\left[x_{1}, \ldots, x_{r}, \xi_{1}, \ldots, \xi_{s} \rrbracket \cong \mathbb{C}\left[x_{1}, \ldots, x_{m}, \xi_{1}, \ldots, \xi_{n}\right]\right.$ then $r=m, n=s$.

Smoothness of a point of a supervariety cannot be checked at the classical level as the next examples show.

Example 3.2. Consider the supervariety $X$ with coordinate ring

$$
\mathbb{C}[X]=\mathbb{C}[x, y, \xi, \eta] /(\xi \eta) .
$$


Its reduced variety is the affine plane, where all the closed points are smooth in the classical sense. It is immediate to check that this supervariety has no smooth points according to Definition 3.1.

Example 3.3. Take the supervariety with coordinate $\operatorname{ring} \mathbb{C}[x, y, \xi, \eta] /(\xi x+\eta y)$. Again its reduced variety is the affine plane. One can check that all (closed) points are smooth except the origin.

Since the notion of smoothness is local we can assume that $X$ is an affine supervariety, with coordinate ring $\mathbb{C}[X]=\mathbb{C}\left[x_{1}, \ldots, x_{m}, \xi_{1}, \ldots, \xi_{n}\right] / I$, where $I=$ $\left(f_{1}, \ldots, f_{p}, \phi_{1}, \ldots, \phi_{q}\right)$. In this case $\mathbb{O}_{X, P}$ is the localization of $\mathbb{C}[X]$ at the point $P$ (see Definition 2.4).

Definition 3.4. As in the classical setting, we define the jacobian of $f_{1}, \ldots, f_{p}$, $\phi_{1}, \ldots, \phi_{q} \in \mathbb{C}\left[x_{1}, \ldots, x_{m}, \xi_{1}, \ldots, \xi_{n}\right]$ at a point $P$ as

$$
\operatorname{Jac}(f, \phi)(P)=\left(\begin{array}{cccccc}
\frac{\partial f_{1}}{\partial x_{1}}(P) & \cdots & \frac{\partial f_{1}}{\partial x_{m}}(P) & \frac{\partial f_{1}}{\partial \xi_{1}}(P) & \cdots & \frac{\partial f_{1}}{\partial \xi_{n}}(P) \\
\vdots & & \vdots & \vdots & \vdots \\
\frac{\partial \phi_{q}}{\partial x_{1}}(P) & \cdots & \frac{\partial \phi_{q}}{\partial x_{m}}(P) & \frac{\partial \phi_{q}}{\partial \xi_{1}}(P) & \cdots & \frac{\partial \phi_{q}}{\partial \xi_{n}}(P)
\end{array}\right)
$$

(for the definition of $\partial f / \partial x$ see for example [Varadarajan 2004]). The rank of the jacobian is given by $a \mid b$ where $a$ and $b$ are the ranks of the $m \times p, n \times q$ diagonal blocks.

Lemma 3.5. Let the notation be as above. Let $P \in|X|$ be a closed point, i.e., a maximal ideal $m_{P}$ in $\mathbb{C}[X]$. Then

$$
\operatorname{rk}(\operatorname{Jac}(f, \phi)(P))=m \mid n-\operatorname{dim}\left(m_{P} / m_{P}^{2}\right) .
$$

Proof. The proof is the same as in ordinary case (see for example [Hartshorne 1977, p. 32]). We sketch it. We have a natural identification

$$
F: M_{P} / M_{P}^{2} \cong \mathbb{C}^{m \mid n}, \quad f \mapsto d f_{P}:=\left(\frac{\partial f}{\partial x_{1}}(P), \ldots, \frac{\partial f}{\partial \xi_{n}}(P)\right),
$$

where $M_{P}$ denotes the maximal ideal corresponding to the point $P$ in $\mathbb{C}\left[x_{1}, \ldots, x_{m}\right.$, $\left.\xi_{1}, \ldots, \xi_{n}\right]$. Viewing the rows of $\operatorname{Jac}(f, \phi)$ as vectors in $\mathbb{C}^{m \mid n}$ the above identification tells us immediately that

$$
\operatorname{rk}(\operatorname{Jac}(f, \phi)(P))=\operatorname{dim}\left(I+M_{P}^{2}\right) / M_{P}^{2}
$$

where $I=\left(f_{1}, \ldots, f_{p}, \phi_{1}, \ldots, \phi_{q}\right)$. Since localizations commute with quotients, we have

$$
m_{P} / m_{P}^{2} \cong\left(M_{P} / I\right) /\left(\left(M_{P}^{2}+I\right) / I\right)=M_{P} /\left(M_{P}^{2}+I\right) .
$$


Hence

$$
\operatorname{rk}(\operatorname{Jac}(f, \phi)(P))=\operatorname{dim}\left(I+M_{P}^{2}\right) / M_{P}^{2}=\operatorname{dim} M_{P} / M_{P}^{2}-\operatorname{dim} M_{P} /\left(M_{P}^{2}+I\right) .
$$

If $A$ is a local superring with maximal ideal $m$, let $\operatorname{Gr}(A)=\bigoplus m^{i} / m^{i+1}$.

Proposition 3.6. Let $P$ be a smooth point of an affine supervariety $X$ with dimension $r \mid s$ in $P$.

(1) $m_{P} / m_{P}^{2}$ has dimension $r \mid s$.

(2) $\operatorname{Gr}\left(\mathscr{O}_{X, P}\right)=\mathbb{C}\left[x_{1}, \ldots, x_{r}, \xi_{1}, \ldots, \xi_{s}\right]$, where $\operatorname{Gr}\left(\mathscr{O}_{X, P}\right)=\oplus_{i} m_{P}^{i} / m_{P}^{i+1}$.

(3) $\operatorname{rk}(\operatorname{Jac}(f, \phi)(P))=m|n-r| s$.

Proof. Statements (1) and (2) follow from Lemma A.5 in the Appendix, and (3) is a consequence of Lemma 3.5.

Remark 3.7. The proof of this result resembles the one for the commutative setting. One difference that may cause confusion is this: When we localize $\mathbb{C}[X]$ to obtain $\mathcal{O}_{X, P}$ we are using a maximal ideal of the even part $\mathbb{C}[X]_{0}$, that is, the ideal generated by $x_{i}-a_{i}$ and $\xi_{i} \xi_{j}$, for all $i$ and $j<i$, where the $a_{i} \in \mathbb{C}$ are the coordinates of the closed point $P$. On the other hand, when we are completing the local superalgebra $\mathcal{O}_{X, P}$ we are taking the inverse limit of the system $\mathcal{O}_{X, P} / m_{P}^{n}$, where $m_{P}$ is the maximal ideal of this superalgebra, hence it is a graded object and it will necessarily contain all the odd generators.

Remark 3.8. If $P$ is smooth and $X$ has dimension $r \mid s$ at $P, m_{P} / m_{P}^{2}$ is generated by $r \mid s$ elements. Hence, by Theorem A.6, $m_{P}$ is generated by $r \mid s$ elements.

Remark 3.9. The affine supervariety $X$ is embedded in $\mathbb{C}^{m \mid n}$ via the chosen explicit presentation of its coordinate ring $\mathbb{C}[X]$. Hence we can give to the set of closed points of $X$ a complex topology inherited from this embedding. However this topology is independent from the embedding; this is a classical fact, still valid in this setting since it is a topological question. We want to show that the closed points of the supervariety $X$ equipped with this complex topology, admit a unique supermanifold structure in a suitable complex neighbourhood $U$ of the smooth point $P$. In other words, we want to show that

$$
\left.\left.\left(\mathscr{H}_{\mathbb{C}^{m \mid n}} / \mathscr{K}\right)\right|_{U} \cong\left(\mathscr{H}_{\mathbb{C}^{m}} / \mathscr{K}^{\mathrm{red}}\right)\right|_{U} \otimes \mathbb{C}\left[\xi_{1}, \ldots, \xi_{s}\right],
$$

where $\mathscr{K}$ is the ideal sheaf whose global sections are generated in $\mathscr{H}_{\mathbb{C}^{m \mid n}}$ by the ideal $I$ of the supervariety $X$. The whole question in the supergeometry setting is to show the existence of a local splitting $(*)$. To settle this problem our strategy is to use the implicit functions theorem, which is still valid in this setting. We recall the statement from [Leites 1980, p. 52]: 
Theorem 3.10. Let $M$ be a complex supermanifold, $P \in|U|$, where $U \subset M$, is isomorphic to an open in $\mathbb{C}^{r \mid s}$. Let $K$ be the ideal in $\mathscr{H}_{M}(U)$ generated by $g_{1}, \ldots, g_{p}, \gamma_{1}, \ldots, \gamma_{q}$ vanishing ${ }^{2}$ at $P$ and with linearly independent differentials at $P$. Then there exists a unique subsupermanifold

$$
N=\left(|N|, \mathscr{H}_{N}\right), \quad \mathscr{H}_{N}=\left.\mathscr{H}_{M}\right|_{U} / \mathscr{K}
$$

where $\mathscr{T}$ is the sheaf of ideals with global sections $K$ and $|N|$ is the topological space whose existence is granted by the classical result.

Remark 3.11. The key for the proof of this result is the fact that any set of functions $g_{1}, \ldots, g_{p}, \gamma_{1}, \ldots, \gamma_{q}$ with linearly independent differentials at $P$ can be completed to obtain a set of local coordinates in a neighborhood of $P$. More details on this can be found in [Varadarajan 2004, p. 148].

This theorem allows us, in a special case, to obtain immediately the result we are after.

Corollary 3.12. Let $P \in|X|$ be a smooth point, and let $X$ have dimension $r \mid s$ at $P$. Let the ideal I of the supervariety $X$ be of the form $\left(f_{1}, \ldots, f_{m-r}, \phi_{1}, \ldots, \phi_{n-s}\right)$ (in this case we say that $X$ is a complete intersection). Then in a neighborhood of $P, X$ admits a complex supermanifold structure (in the sense of Observation 3.9).

Proof. This is a direct application of the Theorem 3.10. $X$ is defined in $\mathbb{C}^{m \mid n}$ by the polynomials $f_{1}, \ldots, f_{m-r}, \phi_{1}, \ldots, \phi_{n-s}$ with $\operatorname{rk}\left(\operatorname{Jac}\left(f_{i}, \phi_{j}\right)(P)\right)=m|n-r| s$. Consider the ideal $K$ generated in $\mathscr{H}\left(\mathbb{C}^{m \mid n}\right)$ by the $f_{i}$ and $\phi_{j}$. Then there exists a unique subsupermanifold $N$ of $\mathbb{C}^{m \mid n}$ such that $\mathscr{H}_{N}=\left.\left(\mathscr{H}_{\mathbb{C}^{m \mid n}} / \mathscr{K}\right)\right|_{U}$ for a suitable neighborhood $U$ of $P, \mathscr{K}$ is the ideal sheaf whose global sections are $K$.

In general the ideal $I$ of the supervariety $X$ is given by $\left(f_{1}, \ldots, f_{p}, \phi_{1}, \ldots, \phi_{q}\right)$ where $p|q>m| n-r \mid s$. We want to show that, as it happens for the classical setting, $X$ is locally a complete intersection, so that we can conclude our discussion with the same reasoning as in Corollary 3.12. Let $P \in X$ be a smooth point and assume $f_{1}, \ldots, f_{m-r}, \phi_{1}, \ldots, \phi_{n-s}$ are such that

$$
\operatorname{rk}\left(\operatorname{Jac}\left(f_{1}, \ldots, f_{m-r}, \phi_{1}, \ldots, \phi_{n-s}\right)(P)\right)=m|n-r| s .
$$

Let $X^{\prime}$ be the variety corresponding to the ring

$$
\mathbb{C}\left[X^{\prime}\right]=\mathbb{C}\left[x_{1}, \ldots, x_{m}, \xi_{1}, \ldots, \xi_{n}\right] /\left(f_{1}, \ldots, f_{m-r}, \phi_{1}, \ldots, \phi_{n-s}\right)
$$

and let $\mathcal{O}_{X^{\prime}, P}$ denote its local ring at the closed point $P$. We are going to show the following:

\footnotetext{
${ }^{2}$ We say that $f \in \mathscr{H}_{M}(U)$ vanishes at $P$ if $f$ is annihilated by the morphism $\mathscr{H}_{M}(U) \rightarrow \mathscr{H}_{M, P} \rightarrow$ $\mathscr{H}_{M, P} / m_{h, P} \cong \mathbb{C}$, where $m_{h, P}$ is the maximal ideal in $\mathscr{H}_{M, P}$.
} 
1. $P$ is a smooth point of $X^{\prime}$, and $X^{\prime}$ has the same dimension as $X$, that is, $\bigcirc_{X^{\prime}, P}=$ $\mathbb{C}\left[\left[x_{1}, \ldots, x_{r}, \xi_{1}, \ldots, \xi_{s}\right]\right]$. This implies that $X^{\prime}$ is a complete intersection.

2. $X$ and $X^{\prime}$ are locally isomorphic: $\mathcal{O}_{X, P} \cong \mathcal{O}_{X^{\prime}, P}$. Since this holds for every point in a neighbourhood of $P$, we have $0_{X}(U) \cong 0_{X^{\prime}}(U)$. Hence we can apply Theorem 3.10 to $X^{\prime}$ to conclude that $X$ admits a supermanifold structure near $P$.

Lemma 3.13. There is a commutative diagram

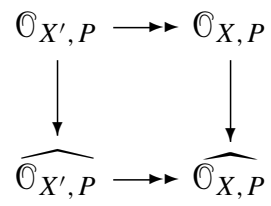

where the orizontal arrows are surjections, while the vertical ones injections.

Proof. Observe that since there is a surjection $\mathbb{C}\left[X^{\prime}\right] \rightarrow \mathbb{C}[X]$ we also have a surjective morphism

$$
\mathrm{O}_{X^{\prime}, P} \rightarrow \mathrm{O}_{X, P}
$$

mapping the maximal ideal onto the maximal ideal (this is a property of localizations). This will give raise to a surjective system

$$
\mathrm{O}_{X^{\prime}, P} / m_{P}^{\prime n} \rightarrow \mathrm{O}_{X, P} / m_{P}^{n}
$$

where $m_{P}$ and $m_{P}^{\prime}$ denote the maximal ideals in $\mathscr{O}_{X, P}$ and $\mathscr{O}_{X^{\prime}, P}$. Hence $\widehat{\mathrm{O}_{X^{\prime}, P}} \rightarrow$ $\widehat{O_{X, P}}$ is a surjective map. The vertical arrows are injections since $\cap m_{P}^{i}=\cap m_{P}^{\prime i}=(0)$. This happens since this is true in the ordinary case and since the odd variables disappear for large $i$.

Remark 3.14. From Lemma 3.5 we see directly that $\operatorname{dim}\left(m_{P} / m_{P}^{2}\right)=\operatorname{dim}\left(m_{P}^{\prime} / m_{P}^{\prime 2}\right)$ and, since the point $P$ is smooth,

$$
\operatorname{dim}\left(m_{P} / m_{P}^{2}\right)=\operatorname{dim}\left(m_{P}^{\prime} / m_{P}^{\prime 2}\right)=r \mid s .
$$

Hence, by Theorem A.6, both $m_{P}$ and $m_{P}^{\prime}$ are generated by $r \mid s$ elements.

\section{Lemma 3.15.}

$$
\widehat{O_{X^{\prime}, P}} \cong \mathbb{C}\left[\left[x_{1}, \ldots, x_{r}, \xi_{1}, \ldots, \xi_{s} \rrbracket / I\right.\right.
$$

for a suitable ideal $I$.

Proof. By the Theorem A.2, there exists a unique map

$$
\left.\mathbb{C}\left[x_{1}, \ldots, x_{r}, \xi_{1}, \ldots, \xi_{s}\right]\right] \widehat{\mathrm{O}_{X^{\prime}, P}}
$$

sending the $x_{i}$ and $\xi_{j}$ into $r \mid s$ generators of the maximal ideal $m_{P}^{\prime}$. So the map is surjective and we obtain our result.

Proposition 3.16. $\widehat{O_{X^{\prime}, P}} \cong \widehat{O_{X, P}}=\mathbb{C} \llbracket x_{1}, \ldots, x_{r}, \xi_{1}, \ldots, \xi_{s} \rrbracket$. 
Proof. By Lemma 3.13,

$$
\widehat{O_{X^{\prime}, P}} / J \cong \widehat{O_{X, P}}=\mathbb{C} \llbracket x_{1}, \ldots, x_{r}, \xi_{1}, \ldots, \xi_{s} \rrbracket
$$

By the Theorem A.4 in the Appendix, we get the result.

We have proved the local isomorphism in the completions, now we turn our attention to the local rings.

Lemma 3.17. $\mathcal{O}_{X^{\prime}, P} \cong \mathcal{O}_{X, P}$.

Proof. By Proposition 3.16 and Lemma 3.13.

This concludes the proof of the following:

Theorem 3.18. Let $X$ be a complex algebraic supervariety, $P$ a smooth point of $X$. Then, there exist a neighbourhood of $P$ where we can give to $X$ a unique structure of a complex supermanifold.

Proof. Assume without loss of generality that $X$ is affine and has dimension $r \mid s$ at $P$. Let

$$
\mathbb{C}[X]=\mathbb{C}\left[x_{1}, \ldots, x_{m}, \xi_{1}, \ldots, \xi_{n}\right] /\left(f_{1}, \ldots, f_{p}, \phi_{1}, \ldots, \phi_{q}\right)
$$

be the coordinate ring of $X$. Let $X^{\prime}$ be the algebraic supervariety defined by the coordinate ring

$$
\mathbb{C}\left[X^{\prime}\right]=\mathbb{C}\left[x_{1}, \ldots, x_{m}, \xi_{1}, \ldots, \xi_{n}\right] /\left(f_{1}, \ldots, f_{m-r}, \phi_{1}, \ldots, \phi_{n-s}\right)
$$

where $\operatorname{rk}\left(\operatorname{Jac}\left(f_{1}, \ldots, f_{m-r}, \phi_{1}, \ldots, \phi_{n-s}\right)(P)\right)=m|n-r| s$. Then by Corollary 3.12 the result holds for $X^{\prime}$ and by Lemma 3.17 $X$ and $X^{\prime}$ are locally isomorphic.

The next lemma will be crucial in the discussion of smoothness of algebraic supergroups in Section 4.

Lemma 3.19. Let $m_{P}$ be generated by $r \mid s$ elements. Then $P$ is smooth if

$$
\operatorname{Gr}\left(O_{X, P}\right)=\mathbb{C}\left[x_{1}, \ldots, x_{r}, \xi_{1}, \ldots, \xi_{s}\right] .
$$

Proof. By Theorem A.2, there exists a surjective map

$$
\mathbb{C} \llbracket x_{1}, \ldots, x_{r}, \xi_{1}, \ldots, \xi_{s} \rrbracket \rightarrow \widehat{\mathrm{O}_{X, P}}
$$

sending $x_{1}, \ldots, x_{r}, \xi_{1}, \ldots, \xi_{s}$ into the generators of the maximal ideal of $\widehat{O_{X, P}}$. Hence $\widehat{O_{X, P}}=\mathbb{C}\left[\left[x_{1}, \ldots, x_{r}, \xi_{1}, \ldots, \xi_{s}\right]\right] / J$ for some ideal $J$. Since $\operatorname{Gr}\left(\widehat{O}_{X, P}\right)=$ $\operatorname{Gr}\left(\widehat{O_{X, P}}\right)$ by Lemma A.5 the result follows by Lemma A.3. 


\section{Smoothness of supergroups}

In this section we want to show that affine algebraic supergroups are smooth, that is all closed points are smooth. In other words we show that the set of closed points of an affine supergroup has a supermanifold structure in the sense of Observation 3.9, hence it is a Lie supergroup. We will do this by using an argument appearing in the classical Cartier's theorem which states that Hopf algebras over a field of characteristic zero are reduced.

It is enough to prove that the identity is a smooth point, since, because of the multiplication law, all closed points have the same local structure.

Let $G$ be an affine algebraic supergroup, $\mathbb{C}[G]$ its Hopf superalgebra with comultiplication $\Delta$, counit $\epsilon$ and antipode $S$. Let $m_{1}=\operatorname{ker} \epsilon$ be the maximal ideal of the identity element and let

$$
m_{1} / m_{1}^{2}=\operatorname{span}_{\mathbb{C}}\left\{t_{1}, \ldots, t_{r+s}\right\}
$$

where $t_{1}, \ldots, t_{r}$ are even and $t_{r+1}, \ldots, t_{r+s}$ are odd.

By an abuse of notation, let $t_{1}, \ldots, t_{r+s}$, denote also the image of these elements modulo $m_{1}^{N}$.

Lemma 4.1. The monomials $t_{1}^{n_{1}} \ldots t_{r+s}^{n_{r+s}}$, with $\sum_{i=1}^{r+s} n_{i}=N$ form a basis for the superspace $m_{1}^{N} / m_{1}^{N+1}$. (Clearly $n_{i}=0,1$ if $i$ is the index of an odd element, $i=r+1, \ldots, s)$.

Proof. The proof is the same as in the classical case, we include it here for completeness; for more details see [Waterhouse 1979, p. 86]. Let $t_{1}^{*}, \ldots, t_{r+s}^{*}$ be the dual basis of $t_{1}, \ldots, t_{r+s}$. Define the map

$$
d_{l}: \mathbb{C}[G]=\mathbb{C} \oplus m_{1} \rightarrow m_{1} / m_{1}^{2} \rightarrow \mathbb{C}
$$

as $d_{l}=t_{l}^{*} \cdot p, l=1, \ldots, r+s$, where $p: \mathbb{C}[G] \rightarrow m_{1} / m_{1}^{2}$ is the natural projection.

Each $d_{l}$ gives rise to a derivation $D_{l}: \mathbb{C}[G] \rightarrow \mathbb{C}[G]$ in the following way:

$$
D_{l}(a):=\sum a^{(1)} d_{l}\left(a^{(2)}\right), \quad \text { where } \quad \Delta(a)=\sum a^{(1)} \otimes a^{(2)} .
$$

Observe that

$$
\epsilon\left(D_{l}(a)\right)=\sum \epsilon\left(a^{(1)}\right) d_{l}\left(a^{(2)}\right)=d_{l} \sum \epsilon\left(a^{(1)}\right) a^{(2)}=d_{l}(a) .
$$

Hence $D_{i}\left(t_{j}\right) \equiv \delta_{i j}$ modulo $m_{1}$ (since $\left.\operatorname{ker} \epsilon=m_{1}\right)$. Let $P\left(T_{1}, \ldots, T_{r+s}\right)$ be a homogeneous polynomial of degree $n$ over $\mathbb{C}$; then

$$
D_{i}(P)\left(t_{1}, \ldots, t_{r+s}\right)=\sum D_{i}\left(t_{j}\right) \frac{\partial P}{\partial T_{j}}\left(t_{1}, \ldots, t_{r+s}\right) .
$$


Since $\partial P / \partial T_{j}\left(t_{1}, \ldots, t_{r+s}\right) \in m_{1}^{n-1}$, we have $D_{i}(P) \equiv \partial P / \partial t_{j}$ modulo $m_{1}^{n}$. Since the congruence $x \equiv y$ modulo $m_{1}^{n}$ implies $D_{i}(x) \equiv D_{i}(y)$ modulo $m_{1}^{n-1}$, we get

$$
D_{r+s}^{n_{r+s}} \ldots D_{1}^{n_{1}} t_{1}^{n_{1}} \ldots t_{r+s}^{n_{r+s}}=n_{1} ! \ldots n_{r+s} ! \quad \bmod m_{1}
$$

while on all other monomials the composition of the $D_{i}$ will give zero. Hence, given a relation $P$ in $m_{1}^{n+1}$, one can single out the coefficient of any monomial by applying the correct sequence of $D_{i}$ 's.

Corollary 4.2. The identity $1 \in|G|$ is a smooth point.

Proof. By Lemma 4.1 and Lemma A.5, the graded associated ring to $\mathscr{O}_{G, 1}$ is

$$
\operatorname{Gr}\left(O_{1, G}\right)=\mathbb{C}\left[t_{1}, \ldots, t_{r}, \theta_{1}, \ldots, \theta_{s}\right] .
$$

This implies by Lemma 3.19 that the identity point is smooth.

Corollary 4.3. If $G$ is an affine supergroup, then $G$ is smooth, that is all its closed points are smooth.

Proof. Let $h_{G}$ denote the functor of points of $G$ and $\mu: h_{G} \times h_{G} \rightarrow h_{G}$ the natural transformation corresponding to the group law. Let $g \in|G|$ be a closed point. $g$ can be identified with an element of $h_{G}(\mathbb{C}) \subset h_{G}(A)$. Hence we can define a natural transformation

$$
l_{g}: h_{G} \rightarrow h_{G}, \quad l_{g, A}(x)=m_{A}(g, x), \forall x \in h_{G}(A)
$$

This natural transformation corresponds to an isomorphism of $G$ into itself, hence $O_{G, 1} \cong O_{G, g}$, so $g$ is smooth. (For more details on the correspondence between natural transformations between functor of points and morphisms of the supervarieties see [Caston and Fioresi 2007] Chapter 3).

\section{The Stabilizer Theorem}

In this section we use the same letter $X$ to denote both a supervariety $X$ and its functor of points $h_{X}$. Let $G$ be an affine algebraic supergroup acting on an affine supervariety $X$, in other words we have a morphism

$$
\rho: G \times X \rightarrow X, \quad(g, x) \mapsto g \cdot x, \quad \forall g \in G(A), x \in X(A)
$$

satisfying the usual properties, viewed in the category of supervarieties. Let $u$ be a topological point of $X$, that is $u \in|X|$ or equivalently $u \in X(\mathbb{C})=\operatorname{Hom}(\mathbb{C}[X], \mathbb{C})$. Let $m_{u}$ be the maximal ideal corresponding to $u$. Notice that $u$ can be viewed naturally as an $A$-point $u_{A}$ for all superalgebras $A$ since $\mathbb{C} \subset A$. So we have a morphism

$$
\tau: G \rightarrow X, \quad g \mapsto g \cdot u_{A}
$$

or, equivalently,

$$
\tilde{\tau}: \mathbb{C}[X] \rightarrow \mathbb{C}[G]
$$


Definition 5.1. We define the stabilizer supergroup functor of a point $u \in|X|$ with respect to the action $\rho$ as the group-valued functor $\operatorname{Stab}_{u}:$ (salg) $\rightarrow$ (sets) given by

$$
\operatorname{Stab}_{u}(A)=\left\{g \in G(A) \quad \mid \quad \tau_{A}(g)=g \cdot u_{A}=u_{A}\right\},
$$

where $\tau_{A}: G(A) \rightarrow X(A)$ or, equivalently,

$$
\operatorname{Stab}_{u}(A)=\left\{g \in G(A)=\operatorname{Hom}(\mathbb{C}[G], A) \quad \mid \quad g \cdot \tilde{\tau}=u_{A}\right\}
$$

We now prove that this functor is representable by an affine supergroup; in the next section we describe some important applications of this result.

Theorem 5.2. Let $G$ be an affine supergroup acting on an affine supervariety $X$ and let $u$ be a topological point of $X$. Then $\mathrm{Stab}_{u}$ is an affine supergroup.

Proof. The stabilizer can be described in an equivalent way as

$$
\operatorname{Stab}_{u}(A)=\left\{g \in G(A)|(g \cdot \tau)|_{m_{u}}=0\right\}
$$

where $m_{u} \subset \mathbb{C}[X]$ is the ideal of $u$. Let $I$ be the ideal in $\mathbb{C}[G]$ generated by $\tilde{\tau}(x)$ for all $x \in m_{u}$. One can immediately check that $g \in G(A)=\operatorname{Hom}(\mathbb{C}[G], A)$ is in $\operatorname{Stab}_{u}(A)$ if and only if $g$ factors via $\mathbb{C}[G] / I$, that is, $g: \mathbb{C}[G] \rightarrow \mathbb{C}[G] / I \rightarrow A$.

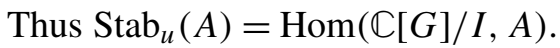

\section{The classical series of Lie supergroups}

In [Kac 1977] Kac proved a classification theorem for simple Lie superalgebras. The description of the supergroup functors, corresponding to the classical superseries of Lie superalgebras introduced by Kac, appeared in [Deligne and Morgan 1999, p. 70]; however no representation statement was proved there.

In this section we describe the supergroup functors corresponding to the classical superseries and show they are representable (that is, they are algebraic supergroups), and hence are Lie supergroups by the results of Section 4. For the series $A(m, n), B(m, n), C(n)$ and $D(m, n)$ this result was proved in [Varadarajan 2004, p. 289] with differential techniques.

One should also prove that the Lie superalgebras ${ }^{3}$ of these Lie supergroups coincide with the classical series mentioned above; however this goes beyond the scope of this paper.

The $\boldsymbol{A}(\boldsymbol{n})$ series. Define $\mathrm{GL}_{m \mid n}(A)$ as the set of all invertible morphisms $g: A^{m \mid n} \rightarrow$ $A^{m \mid n}$. This is equivalent to asking that the Berezinian or superdeterminant

$$
\operatorname{Ber}(g)=\operatorname{Ber}\left(\begin{array}{ll}
p & q \\
r & s
\end{array}\right)=\operatorname{det}\left(p-q s^{-1} r\right) \operatorname{det} s^{-1}
$$

\footnotetext{
${ }^{3}$ For the definition of Lie superalgebra of an algebraic supergroup, see [Caston and Fioresi 2007, Chapter 5].
} 
(see [Berezin 1987]) be invertible in $A$, where $p$ and $s$ are $m \times m, n \times n$ matrices of even elements in $A$, while $q$ and $r$ are $m \times n, n \times m$ matrices of odd elements in $A$. A necessary and sufficient condition for $g \in \mathrm{GL}_{m \mid n}(A)$ to be invertible is that $p$ and $s$ are invertible. The group-valued functor

$$
\mathrm{GL}_{m \mid n}:(\mathrm{salg}) \rightarrow \text { (sets), } \quad A \rightarrow \mathrm{GL}_{m \mid n}(A)
$$

is an affine supergroup called the general linear supergroup and is represented by the algebra $\mathbb{C}\left[\mathrm{GL}_{m \mid n}\right]:=\mathbb{C}\left[x_{i j}, y_{\alpha \beta}, \xi_{i \beta}, \gamma_{\alpha j}, z, w\right] /((w \operatorname{det} x-1, z \operatorname{det} y-1)$, where $i, j=1, \ldots m$ and $\alpha, \beta=1, \ldots n$.

Consider the morphism

$$
\rho: \mathrm{GL}_{m \mid n} \times \mathbb{C}^{1 \mid 0} \rightarrow \mathbb{C}^{1 \mid 0}, \quad(g, c) \rightarrow \operatorname{Ber}(g) c .
$$

The stabilizer of the point $1 \in \mathbb{C}^{1 \mid 0}$ coincides with $\operatorname{SL}_{m \mid n}(A)$, the special linear supergroup, defined as the matrices in $\mathrm{GL}_{m \mid n}(A)$ with Berezinian 1. By Theorem 5.2, $\mathrm{SL}_{m \mid n}$ is representable, and by Section 4 it is a complex supermanifold. Moreover one can check that $A(m, n)=\operatorname{Lie}\left(\mathrm{SL}_{m \mid n}\right)$.

The $B(m, n), C(n)$ and $D(m, n)$ series. Consider the morphism

$$
\rho: \mathrm{GL}_{m \mid 2 n} \times \mathscr{B} \rightarrow \mathscr{B} \quad(g, \psi(\cdot, \cdot)) \rightarrow \psi(g \cdot, g \cdot),
$$

where $\mathscr{B}$ is the vector superspace of all the symmetric bilinear forms on $\mathbb{C}^{m \mid 2 n}$. The stabilizer of the point $\Phi$ the standard bilinear form on $\mathbb{C}^{m \mid 2 n}$ is the supergroup functor $\operatorname{Osp}_{m \mid 2 n}$. Again this is an algebraic supergroup by Theorem 5.2 and it is also a complex supermanifold. One can check that $B(m, n)=\operatorname{Lie}\left(\operatorname{Osp}_{2 m+1 \mid 2 n}\right)$, $C(n)=\operatorname{Lie}\left(\mathrm{Osp}_{2 \mid 2 n-2}\right)$ and $D(m, n)=\operatorname{Lie}\left(\mathrm{Osp}_{2 m \mid 2 n}\right)$.

The $\boldsymbol{P}(\boldsymbol{n})$ series. Define the algebraic supergroup $\pi \mathrm{Sp}_{n \mid n}$ as we did for $\operatorname{Osp}_{m \mid n}$, by taking antisymmetric bilinear forms instead of symmetric ones. Consider the action

$$
\pi \mathrm{Sp}_{n \mid n} \times \mathbb{C}^{1 \mid 0} \rightarrow \mathbb{C}^{1 \mid 0}, \quad(g, c) \mapsto \operatorname{Ber}(g) c .
$$

By Theorem 5.2, Stab $_{1}$ is an affine algebraic supergroup, hence a Lie supergroup. It is corresponding to the $P(n)$ series.

The $Q(n)$ series. Let $D=\mathbb{C}[\eta] /\left(\eta^{2}+1\right)$. This is a noncommutative superalgebra. Define the supergroup functor $\mathrm{GL}_{n}(D):(\mathrm{salg}) \rightarrow$ (sets), with $\mathrm{GL}_{n}(D)(A)$ the group of automorphisms of the left supermodule $A \otimes D$. In [Deligne and Morgan $1999]$ is proven the existence of a morphism called the odd determinant

$$
\text { odet }: \mathrm{GL}_{n}(D) \rightarrow \mathbb{C}^{0 \mid 1} \text {. }
$$

Reasoning as before, define

$$
\mathrm{GL}_{n}(D) \times \mathbb{C}^{0 \mid 1} \rightarrow \mathbb{C}^{0 \mid 1}, \quad g, c \rightarrow \operatorname{odet}(g) c .
$$


Then $G=\mathrm{Stab}_{1}$ is an affine algebraic supergroup and for $n \geq 2$ we define $Q g(n)$ as the quotient of $G$ and the diagonal subgroup $\mathrm{GL}_{1 \mid 0}$. This is an algebraic and Lie supergroup and its Lie superalgebra is $Q(n)$.

\section{Appendix: Commutative superalgebra}

Let $k$ be the ground field. We assume all superalgebras to be commutative. As before, we denote with latin letters the even elements and with greek letters the odd elements of a superalgebra.

Theorem A.1. Let A be a finitely generated superalgebra. There exists a unique superalgebra morphism

$$
\phi: k\left[x_{1}, \ldots, x_{m}, \xi_{1}, \ldots, \xi_{n}\right] \rightarrow A,
$$

(where $k\left[x_{1}, \ldots, x_{m}, \xi_{1}, \ldots, \xi_{n}\right]$ denotes the polynomial superalgebra with even indeterminates $x_{i}$ and odd indeterminates $\xi_{j}$ ) sending the $x_{i}$ and the $\xi_{j}$ to chosen elements in A of the correct parity.

This comes from the universality of the construction of the polynomial superalgebra as it is done for example in [Deligne and Morgan 1999, p. 49].

Theorem A.2. Let $A$ be a finitely generated superalgebra and let $\hat{A}=\lim \longleftarrow A / n^{i}$, be its completion with respect an ideal $n$. Let $\hat{n}$ be the ideal in $\hat{A}$ corresponding to $n$. There exists a unique superalgebra morphism $\phi: k\left[\left[x_{1}, \ldots, x_{m}, \xi_{1}, \ldots, \xi_{n}\right]\right] \rightarrow$ $\hat{A}$ sending the $x_{i}$ and the $\xi_{j}$ to chosen elements in $\hat{n}$ of the correct parity.

Proof. This is the same as [Eisenbud 1995, p. 200, Theorem 7.16]. Let's briefly recall it. By Theorem A.1, there is a unique map $k\left[x_{1}, \ldots, x_{m}, \xi_{1}, \ldots, \xi_{n}\right] \rightarrow \hat{A} / \hat{n}^{i}$ sending the $x_{i}$ and the $\xi_{j}$ to chosen elements in $n$. Clearly this maps factors as

$$
\begin{aligned}
& k\left[x_{1}, \ldots, x_{m}, \xi_{1}, \ldots, \xi_{n}\right] \rightarrow \\
& k\left[x_{1}, \ldots, x_{m}, \xi_{1}, \ldots, \xi_{n}\right] /\left(x_{1}, \ldots, x_{m}, \xi_{1}, \ldots, \xi_{n}\right)^{i} \rightarrow \hat{A} / \hat{n}^{i} .
\end{aligned}
$$

One can check that

$$
\frac{k\left[x_{1}, \ldots, x_{m}, \xi_{1}, \ldots, \xi_{n}\right]}{\left(x_{1}, \ldots, x_{m}, \xi_{1}, \ldots, \xi_{n}\right)^{i}} \cong \frac{k\left[\left[x_{1}, \ldots, x_{m}, \xi_{1}, \ldots, \xi_{n}\right]\right]}{\left(x_{1}, \ldots, x_{m}, \xi_{1}, \ldots, \xi_{n}\right)^{i}}
$$

hence by the universal property of the inverse limit we have obtained the required map and its uniqueness.

Lemma A.3. $I=(0)$ if

$$
\operatorname{Gr}\left(k \llbracket x_{1}, \ldots, x_{r}, \xi_{1}, \ldots, \xi_{s} \rrbracket / I\right) \cong \operatorname{Gr}\left(k \llbracket\left[x_{1}, \ldots, x_{r}, \xi_{1}, \ldots, \xi_{s} \rrbracket\right) .\right.
$$


Proof. Let $m$ be the maximal ideal in $k \llbracket x_{1}, \ldots, x_{r}, \xi_{1}, \ldots, \xi_{s} \rrbracket$. There exist $i$ such that $I \subset m^{i}$ but $I \not \subset m^{i+1}$ otherwise we are done since $I \subset \cap m^{i}=(0)$. Then

$$
\left(m^{i} / I\right) /\left(m^{i+1}+I / I\right)=m^{i} /\left(m^{i+1}+I\right) \neq m^{i} / m^{i+1},
$$

which gives a contradiction.

Theorem A.4. $I=(0)$ if

$$
k \llbracket x_{1}, \ldots, x_{r}, \xi_{1}, \ldots, \xi_{s} \rrbracket / I \cong k \llbracket\left[x_{1}, \ldots, x_{r}, \xi_{1}, \ldots, \xi_{s} \rrbracket .\right.
$$

Proof. This is a consequence of Lemma A.3.

Lemma A.5. Let $A$ be a commutative superalgebra and $m$ a maximal ideal. Let $A_{m}$ be the localization of $A$ into the even part $m_{0}$ of the maximal ideal $m$ and $\widehat{A}_{m}$ the completion of $A_{m}$ with respect to the maximal ideal $\tilde{m}$ in $A_{m}$. Then

$$
m^{i} / m^{i+1} \cong \tilde{m}^{i} / \tilde{m}^{i+1} \cong \widehat{m}^{i} / \widehat{m}^{i+1} .
$$

Proof. This is the same as in the commutative case, because localization and completion commute with quotients.

Theorem A.6 (Super-Nakayama's Lemma). Let A be a local commutative superring with maximal (homogeneous) ideal $\mathfrak{m}$. Let $E$ be a finitely generated module for the ungraded ring $A$.

(i) If $\mathfrak{m} E=E$, then $E=0$; more generally, if $H$ is a submodule of $E$ such that $E=\mathfrak{m} E+H$, then $E=H$.

(ii) Let $\left(v_{i}\right)_{1 \leq i \leq p}$ be a basis for the $k$-vector space $E / \mathfrak{m} E$ where $k=A / \mathfrak{m}$. Let $e_{i} \in E$ be above $v_{i}$. Then the $e_{i}$ generate $E$. If $E$ is a supermodule for the superring $A$, and $v_{i}$ are homogeneous elements of the vector superspace $E / \mathfrak{m} E$, we can choose the $e_{i}$ to be homogeneous too (and hence of the same parity as the $v_{i}$ ).

(iii) Suppose $E$ is projective, that is, there is a A-module $F$ such that $E \oplus F=A^{N}$ where $A^{N}$ is the free module for the ungraded ring A of rank $N$. Then $E$ (and hence $F$ ) is free, and the $e_{i}$ above form a basis for $E$.

Proof. See [Caston and Fioresi 2007, Appendix].

Acknowledgements. We thank Prof. V. S. Varadarajan, Dr. L. Caston, Dr. C. Carmeli, Prof. D. Gieseker and Prof. M. Duflo for helpful comments.

\section{References}

[Batchelor 1984] M. Batchelor, "Graded manifolds and supermanifolds", pp. 91-133 in Mathematical aspects of superspace (Hamburg, 1983), NATO Adv. Sci. Inst. Ser. C Math. Phys. Sci. 132, Reidel, Dordrecht, 1984. MR 86m:58018 Zbl 0539.53058 
[Batchelor 1985] M. Batchelor, "Graded manifolds and vector bundles: a functorial correspondence”, J. Math. Phys. 26:7 (1985), 1578-1582. MR 87a:58019 Zbl 0578.57008

[Berezin 1987] F. A. Berezin, Introduction to superanalysis, Math. Physics and Applied Math. 9, D. Reidel, Dordrecht, 1987. MR 89b:58006 Zbl 0659.58001

[Caston and Fioresi 2007] L. Caston and R. Fioresi, "Mathematical foundation of supersymmetry", preprint, 2007. arXiv 0710.5742

[Deligne and Morgan 1999] P. Deligne and J. W. Morgan, "Notes on supersymmetry (following Joseph Bernstein)", pp. 41-97 in Quantum fields and strings: a course for mathematicians (Princeton, 1996/1997), vol. 1, edited by P. Deligne, American Mathematical Society, Providence, RI, 1999. MR 2001g:58007 Zbl 01735158

[Eisenbud 1995] D. Eisenbud, Commutative algebra with a view toward algebraic geometry, Graduate Texts in Math. 150, Springer, New York, 1995. MR 97a:13001 Zbl 0819.13001

[Gruson 1994] C. Gruson, "Description de certains super groupes classiques", Ann. Inst. Fourier (Grenoble) 44:1 (1994), 39-63. MR 95a:17012 Zbl 0792.14001

[Hartshorne 1977] R. Hartshorne, Algebraic geometry, Graduate Texts in Math. 52, Springer, New York, 1977. MR 57 \#3116 Zbl 0367.14001

[Kac 1977] V. G. Kac, “Lie superalgebras", Advances in Math. 26:1 (1977), 8-96. MR 58 \#5803 Zbl 0366.17012

[Kostant 1977] B. Kostant, "Graded manifolds, graded Lie theory, and prequantization”, pp. 177306 in Differential geometrical methods in mathematical physics (Bonn, 1975), edited by K. Bleuler and A. Reetz, Lecture Notes in Math. 570, Springer, Berlin, 1977. MR 58 \#28326 Zbl 0358.53024

[Leites 1980] D. A. Leites, "Introduction to the theory of supermanifolds", Uspekhi Mat. Nauk 35:1 (1980), 3-57. In Russian; translated in Russian Math. Surv. 35:1 (1980), 1-64. MR 81j:58003 Zbl 0462.58002

[Manin 1988] Y. I. Manin, Gauge field theory and complex geometry, Grundlehren der Math. Wissenschaften 289, Springer, Berlin, 1988. MR 89d:32001 Zbl 0641.53001

[Varadarajan 2004] V. S. Varadarajan, Supersymmetry for mathematicians: an introduction, Courant Lecture Notes in Mathematics 11, Courant Institute of Mathematical Sciences, New York, 2004. MR 2005g:58011 Zbl 02123108

[Waterhouse 1979] W. C. Waterhouse, Introduction to affine group schemes, Graduate Texts in Math. 66, Springer, New York, 1979. MR 82e:14003 Zbl 0442.14017

Received March 21, 2007.

\author{
RITA FIORESI \\ DiPARTIMENTO Di MATEMATICA \\ UNIVERSITÀ DI BOLOGNA \\ PIAZZA PORTA SAN DONATO 5 \\ 40126 BOLOGNA \\ ITALY
}

fioresi@dm.unibo.it 